\title{
Emergence of meropenem/vaborbactam resistance in KPC-producing Klebsiella pneumoniae causing bloodstream infections in northern Italy, 2018
}

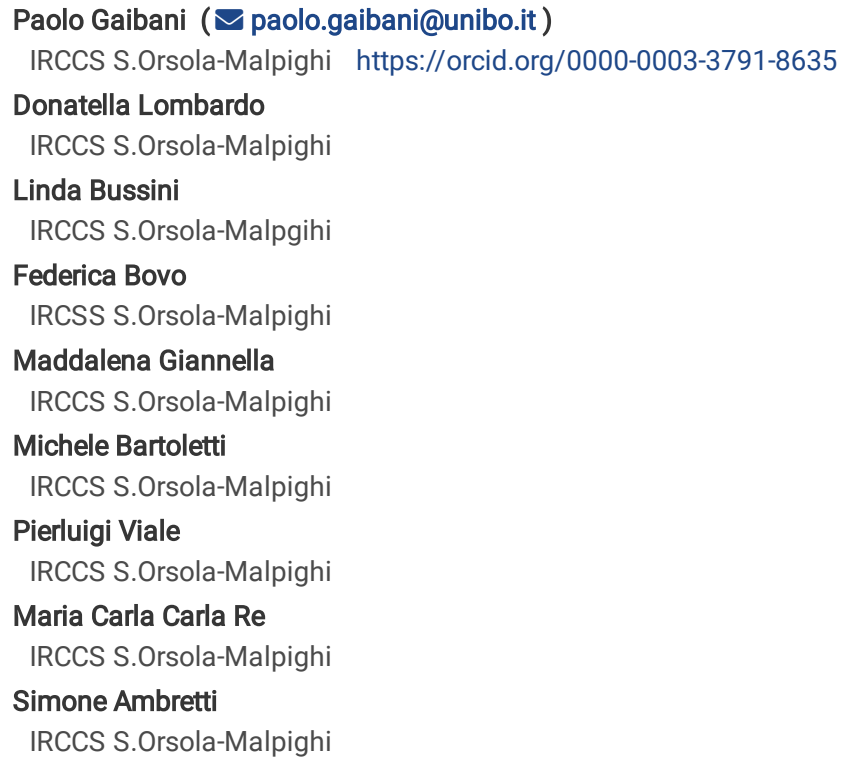




\section{Abstract}

We aimed to investigate the incidence of meropenem/vaborbactam-resistance among KPC-producing Klebsiella pneumoniae (KPC-Kp) bloodstream infection in a large Italian hospital. Meropenem/vaborbactam-resistance was found in $8 \%(n=5)$ KPC-Kp, while $5 \%(n=3)$ strains exhibited cross-resistance to ceftazidime/avibactam. Genomic analysis revealed that meropenem/vaborbactam-resistance was associated to non-functional OmpK35 and OmpK36 porins and no specific mutation was associated to cross-resistance. No specific antimicrobial treatment was related to favorable clinical outcome, while crossresistance was not associated to higher clinical and/or microbiological failures. Our study indicated that resistance to meropenem/vaborbactam was due to porins deficiency and is associated to reduced susceptibility both to ceftazidime/avibactam and carbapenems.

\section{Introduction}

Management and treatment of patients with infections due to Klebsiella pneumoniae carbapenemase (KPC)-producing K. pneumoniae (KPC-Kp) is a daily challenge in clinical practice. In the last decade, the treatment of serious KPC-kp infections was frequently based on the combination of last-resort agents associated with nephrotoxicity, sub therapeutic concentrations, and/or intermediate in vitro activity due to limited options [1].

On 20th November 2018 European Medicines Agency (EMA) approved meropenem/vaborbactam (MEM/VAB) for clinical use in adult patients with difficult to treat carbapenem-resistant Enterobacterales (CRE) infections, satisfying an important medical issue linked to antibiotic-resistant Enterobacterales

(https://www.ema.europa.eu/en/medicines/human/EPAR/vaborem). MEM/VAB represents a novel antibiotic combination therapy, approved for clinical use in Europe indicated for complicated urinary tract infection (cUTI), complicated intra-abdominal infections (cIAl), hospital- acquired pneumonia (HAP), ventilatoracquired pneumonia (VAP) and bacteremia associated with these infections [2].

In vitro studies demonstrated excellent activity of MEM/VAB against KPC-producers with a low ability to acquire resistance [3]. Recent clinical study showed that MEM/VAB had a superior rate of clinical cure against CRE infections in patients with bacteremia, compared to other available therapies [2]. However, limited information is still available on the efficacy of MEM/VAB in clinical practice and the in vivo evolution of emerging resistant strains [3]. Recently, in vitro studies demonstrated that mutations causing loss-of-function of OmpK35 and OmpK36 porins or reduced production can generate resistance to MEM/VAB and/or ceftazidime/avibactam (CAZ/AVI) $[4,5]$.

Aim of this study was to investigate the epidemiology of meropenem/vaborbactam resistance among KPC-Kp strains isolated from patients with bloodstream infection (BSI) and characterize genomically MEM/VAB-resistant strains.

\section{Materials And Methods}

Between 1st January to 31st December 2018, we collected all KPC-producing K. pneumoniae isolated from patients with BSIs hospitalized in the large tertiarycare university hospital in Bologna. The study was conducted in accordance with the Declaration of Helsinki. Analysis was performed with anonymized database. Bacteria was identified by MALDI-TOF (Bruker Daltonics, Germay) and antimicrobial susceptibility testing was performed by MicroScan Walkaway system and confirmed by MIC test strip (Liofilchem, Italy). The MIC value for colistin was determined by reference broth microdilution method [4]. MIC results were interpreted according to EUCAST clinical breakpoints v11.0 (http://www.eucast.org/clinical_breakpoints/). Carbapenemase production was determined by MALDI-TOF and/or NG-Test CARBA 5 (NG Biotech, France), as previously described [6]. Molecular assay was performed to identify carbapenemase gene (Xpert Carba-R, Cepheid).

Whole-genome sequencing of KPC-producing K. pneumoniae (KPC-Kp) strains was performed to identify the molecular mechanism at the basis of meropenem/vaborbactam-resistance as previous described [7]. Briefly, libraries were prepared by the Nextera DNA flex sample preparation kit and sequenced using the Illumina Iseq100 platform (Illumina, San Diego, USA) with a 2x150 paired end run. All read sets were evaluated by FastQC software and then assembled with SPAdes v.3.10 with careful settings. Assembled genomes were screened for Sequence type, known antimicrobial resistance, Sequence type (ST) by CGE server (https://cge.cbs.dtu.dk/services/). Porin genes and Tn4401 isoform were manually investigated by BLAST analysis. A core genome single nucleotide polymorphism (SNP) phylogeny was generated using Parsnp software [8] using draft genomes of KPC-producing K. pneumoniae CC258 strains isolated in Italy, as previously described [8]. Single nucleotide polymorphisms (SNPs) and insertion-deletions (Indels) between MEM/VAB-resistant KPC-Kp genomes were investigated using Breseq [9].

Clinical outcome was defined as all-cause mortality at day 30 after BSI onset (index BC collection day). Microbiologic failure was defined as isolation of KPCKp strain with similar antimicrobial susceptibility pattern following 30 days of target antimicrobial treatment.

\section{Results}

During the study period, in vitro activity of MEM/VAB has been evaluated against 62 KPC-Kp strains isolated from patients with BSIs. The KPC-Kp distribution revealed that $45.2 \%$ of bacteremic patients were hospitalized in intensive care units (ICUs), $34 \%$ in medicine, $9.7 \%$ in haemato-oncology, $6.4 \%$ in surgical and $4.7 \%$ in transplantation wards.

According to EUCAST breakpoints, 54 out of 62 strains (87\%) were susceptible to meropenem/vaborbactam with a median MIC of $0.5 \mathrm{mg} / \mathrm{L}$ (interquartile range [IQR] 0,25-1). On opposite, eight KPC-Kp were resistant to meropenem/vaborbactam by exhibiting a median MIC of 256 mg/L (IQR 64-256), as shown in Table 1. Of note, three out of eight (37.5\%) meropenem/vaborbactam-resistant KPC-Kp strains were also resistant to ceftazidime/avibactam (median MIC 32 , IQR 32-256), while others meropenem/vaborbactam-resistant strains showed a CAZ/AVI MIC equal to $8 \mathrm{mg} / \mathrm{L}$ (Table 1). 
Genomic analysis showed that meropenem/vaborbactam-resistant strains belonged to Clonal Complex 258 (ST1519, ST258 and ST512) and shared similar genetic resistance determinants responsible for resistance to ß-lactams, aminoglycoside, fluoroquinolone and fosfomycin (Table 1). In particular, analysis of ß-lactam resistance genes showed that all isolates shared wild-type bla $a_{\mathrm{KPC}-3}$ gene, while 5 out of 8 carried $b / a_{\mathrm{SHV}-182}$ variant. In addition, environment of $b_{\text {bPC-3 }}$ gene demonstrated that all three KPC-Kp strains harbored Tn4401 isoform a. Genetic analysis of porin genes showed that all meropenem/vaborbactam-resistant KPC-Kp strains had truncated OmpK35 and the insertion of glycine and aspartic acid within OmpK36 at position 134-135 (GD134-135).

Based on genome alignment, a total of 234 SNPs in KpBO3, 336 in KpBO6, 137 in KpBO7, 137 in KpBO8, 115 in KpBO11, 111 in KpBO12, 156 in KpBO13 and 110 in KpB014 were observed against genome of the KpBO3 strain as reference. Comparative analysis of single nucleotide polymorphisms (SNPs) and insertion-deletions (Indels) between MEM/VAB-resistant KPC-Kp strains showed that most SNPs were located intergenic regions or in hypothetical proteins and that no significant difference between MEM/VAB-resistant strains and cross-resistant (i.e. MEM/VAB and CAZ/AVI-resistant) KPC-Kp strains were observed in genes encoding antimicrobial resistance. In addition, phylogenetic analysis showed that genomes of KPC-Kp strains resistant to meropenem/vaborbactam clustered into a single clade (Figure S1, Supplementary data).

Retrospective clinical analysis showed that patients with BSI due to KPC-Kp resistant both to ceftazidime/avibactam and meropenem/vaborbactam had short time of CPE colonization (median 4 days, IQR 0-7). Clinical characteristics of patients with meropenem/vaborbactam-resistant KPC-Kp are shown in Table 2. The 30 -days mortality rates were $100 \%(3 / 3)$ for patients with SOFA score between $10-20,50 \%(1 / 2)$ for patients with score ranging from 5 to 10 and $0 \%(0 / 3)$ for patients below 4 .

Clinical success was achieved in $50 \%$ (4/8) of patients. None of patients were treated with meropenem/vaborbactam-based treatment. Of note, clinical failure occurred for different antimicrobial treatments and in 33\% (1/3) of meropenem/vaborbactam and ceftazidime/avibactam -resistant KPC-Kp strains. In particular, no relapsing infection was observed for all antimicrobial combination treatment. At the same time, microbiological failure was achieved in one patient treated with colistin-meropenem therapy due to persistent bacteremia.

\section{Discussion}

In this study, we evaluated the activities of meropenem/vaborbactam against $62 \mathrm{KPC}-\mathrm{Kp}$ clinical strains collected from bacteremic patients during 2018 . Our findings showed that resistance to meropenem/vaborbactam emerged in 13\% of KPC-Kp strains isolated from bloodstream infection. Of note, meropenem/vaborbactam resistance was associated to high level of MICs for ceftazidime/avibactam. In particular, cross-resistance to ceftazidime/avibactam and meropenem/vaborbactam was observed in three KPC-Kp strains which exhibited high levels of MICs for both ceftazidime/avibactam, meropenem/vaborbactam and carbapenems.

Recent study showed that the selection of KPC-Kp mutants with reduced susceptibility to meropenem-vaborbactam was due to porin mutations associated to increase in the blaKPC gene copy number, whereas was not asccoiated to mutations in the coding region of blaKPC gene [10]. Our results are in agreement with these findings as we found that meropenem/vaborbactam-resistance was due to OmpK35 and OmpK36 porins depletion in our KPC-Kp strains and no specific mutations were observed in bla $\mathrm{KPC}_{\mathrm{C}}$ gene. At the same time, our results showed that no specific mutations were observed in KPC-Kp isolates resistant both to meropenem/vaborbactam and ceftazidime/avibactam in comparison to strains resistant to meropenem/vaborbactam alone. These results are in accordance with previous findings that showed as cross-resistance to meropenem/vaborbactam and ceftazidime/avibactam was due to loss-of-function mutations in the OmpK35 and OmpK36 porins which are also associated to elevated MICs for ß-lactams [4,5].

We observed that patients with BSI due to KPC-Kp resistant both to meropenem/vaborbactam and ceftazidime/avibactam had short colonization time, thus suggesting that cross-resistance did not result from selection pressure due to prolonged antimicrobial therapy while can be plausible that patients acquired cross-resistant strains during hospitalization.

Overall clinical and microbiological failure was observed in two out of three patients with pneumonia, while no specific treatment was associated to clinical success. At the same time, microbiological failure was observed only in a colistin-meropenem combination treatment. Of note, cross-resistance to meropenem/vaborbactam and ceftazidime/avibactam was not associated to clinical or microbiological failure.

The study is limited by the small number of patients with BSI due to meropenem/vaborbactam-resistant strains treated with different combination treatments. Further study is mandatory to evaluate the clinical outcome of different antimicrobial therapies against BSI due meropenem/vaborbactam and/or ceftazidime/avibactam - resistant strains.

In conclusion, here we described the emergence of meropenem/vaborbactam resistance among KPC-Kp strains isolated from bacteremic patients. Further studies are planned to characterize specific-mutations emerging in vivo under different antimicrobial therapy and evaluate the in vitro activity of novel antimicrobials (i.e. cefiderocol, imipenem/cilastatin/relebactam) against meropenem/vaborbactam and/or ceftazidime/avibactam-resistant strains.

\section{Declarations}

\section{Funding}

This work was supported by Italian Ministry of Health (Ricerca Finalizzata, Giovani Ricercatori, GR-2018-12367572).

\section{Conflicts of interest/Competing interests}


The authors declare that they have no conflict of interest.

\section{Availability of data and material}

The data are available for scientists having interest.

\section{Code availability}

Not applicable

\section{Ethics approval}

The study was conducted in the context of normal clinical routine. The study was conducted in accordance with the Declaration of Helsinki. Samples were coded and analysis was performed with anonymized database.

\section{Consent to participate}

Not applicable

\section{Consent for publication}

Not applicable

\section{References}

1. Giacobbe DR, Maraolo AE, Viscoli C (2017) Pitfalls of defining combination therapy for carbapenem-resistant Enterobacteriaceae in observational studies. Eur J Clin Microbiol Infect Dis 36:1707-09. https://doi.org/10.1007/s10096-017-3010-z

2. Wunderink RG, Giamarellos-Bourboulis EJ, Rahav G, Mathers AJ, Bassetti M, Vazquez J, et al (2018) Effect and Safety of Meropenem-Vaborbactam versus Best-Available Therapy in Patients with Carbapenem-Resistant Enterobacteriaceae Infections: The TANGO II Randomized Clinical Trial. Infect Dis Ther 7:439-55. https://doi.org/10.1007/s40121-018-0214-1

3. Lapuebla A, Abdallah M, Olafisoye O, Cortes C, Urban C, Quale J, Landman D (2015) Activity of meropenem combined with RPX7009, a novel $\beta$-lactamase inhibitor, against Gram-negative clinical isolates in New York City. Antimicrob Agents Chemother 59:4856-60. https://doi.org/10.1128/aac.00843-15

4. Gaibani P, Re MC, Campoli C, Viale PL, Ambretti S (2020) Bloodstream infection caused by KPC-producing Klebsiella pneumoniae resistant to ceftazidime/avibactam: epidemiology and genomic characterization. Clin Microbiol Infect 26:516.e1-516.e4. https://doi.org/10.1016/j.cmi.2019.11.011

5. Dulyayangkul P, Wan Nur Ismah WAK, Douglas EJA, Avison MB (2020) Mutation of kvrA Causes OmpK35 and OmpK36 Porin Downregulation and Reduced Meropenem-Vaborbactam Susceptibility in KPC-Producing Klebsiella pneumoniae. Antimicrob Agents Chemother 64:e02208-19. https://doi.org/10.1128/aac.02208-19

6. Foschi C, Gaibani P, Lombardo D, Re MC, Ambretti S (2020) Rectal screening for carbapenemase-producing Enterobacteriaceae: a proposed workflow. J Glob Antimicrob Resist 21:86-90. https://doi.org/10.1016/j.jgar.2019.10.012

7. Gaibani P, Campoli C, Lewis RE, Volpe SL, Scaltriti E, Giannella M et al (2018) In vivo evolution of resistant subpopulations of KPC-producing Klebsiella pneumoniae during ceftazidime/avibactam treatment. J Antimicrob Chemother 73:1525-29. https://doi.org/10.1093/jac/dky082

8. Treangen TJ, Ondov BD, Koren S, Phillippy AM (2014) The Harvest suite for rapid core-genome alignment and visualization of thousands of intraspecific microbial genomes. Genome Biol 15:524. https://doi.org/10.1186/s13059-014-0524-x

9. Barrick JE, Colburn G, Deatherage DE, Traverse CC, Strand MD, Borges JJ et al (2014) Identifying structural variation in haploid microbial genomes from short-read resequencing data using breseq. BMC Genomics 15:1039. https://doi.org/10.1186/1471-2164-15-1039

10. Sun D, Rubio-Aparicio D, Nelson K, Dudley MN, Lomovskaya O (2017) Meropenem- Vaborbactam Resistance Selection, Resistance Prevention, and Molecular Mechanisms in Mutants of KPC-Producing Klebsiella pneumoniae. Antimicrob Agents Chemother 61:e01694-17.

https://doi.org/10.1128/aac.01694-17

\section{Tables}

Table 1. Phenotypic and genotypic characteristics of meropenem/vaborbactam-resistant KPC-producing Klebsiella pneumoniae strains 


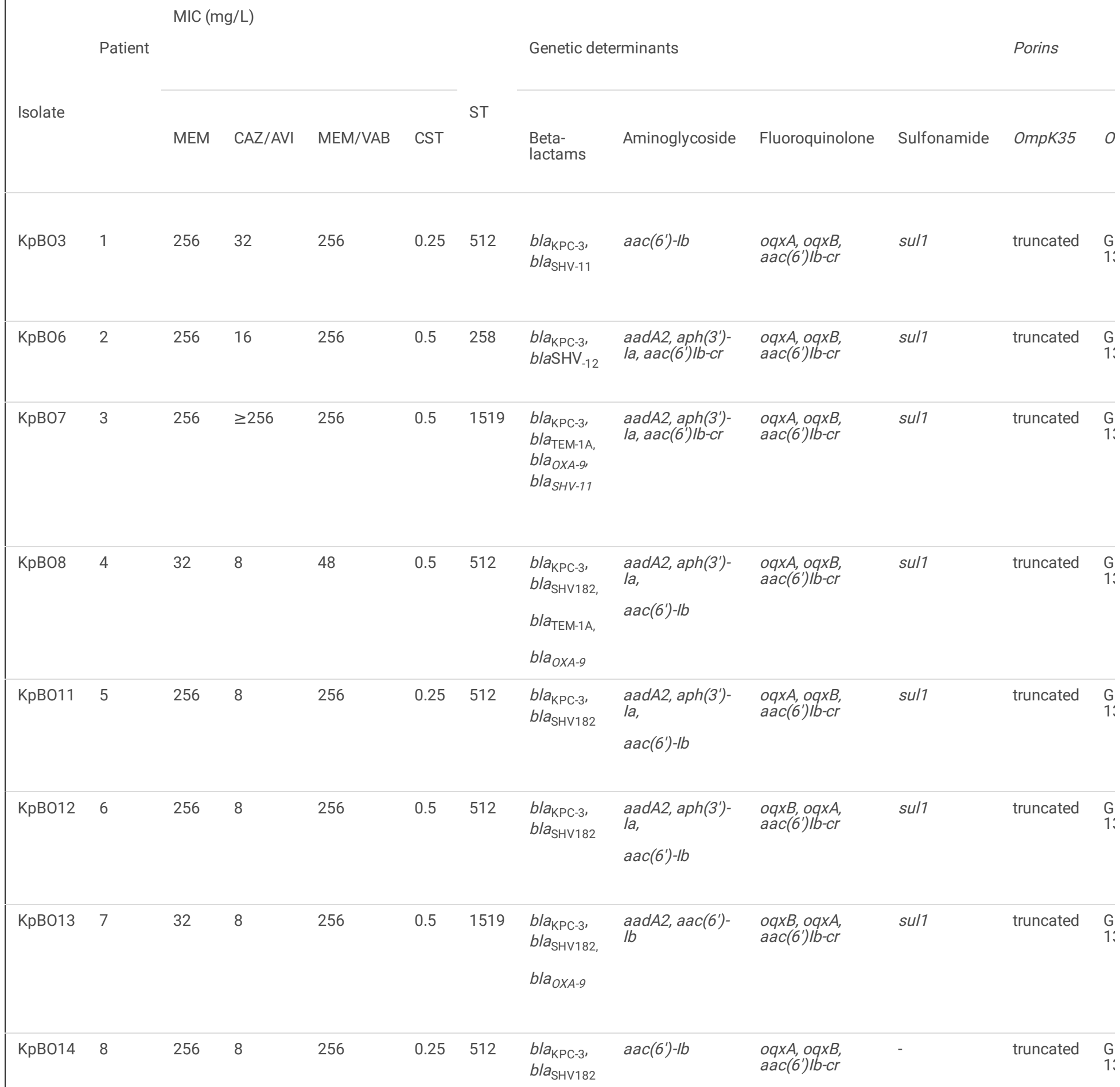

Abbreviations: MEM, meropenem; CAZ/AVI, ceftazidime/avibactam; MEM/VAB, meropenem/vaborbactam; CST, colistin; ST, sequence type.

Table 2. Clinical characteristics of patients with bloodstream infection due to Meropenem/Vaborbactam-resistant KPC-producing Klebsiella pneumoniae (KPCKp) strains 


\begin{tabular}{|c|c|c|c|c|c|c|c|c|c|c|}
\hline Patient & Isolate & $\begin{array}{l}\text { Colonization } \\
\text { days' prior } \\
\text { infection }\end{array}$ & SOFA & $\begin{array}{l}\text { Initial } \\
\text { infection }\end{array}$ & $\begin{array}{l}\text { Previous } \\
\text { Treatment } \\
\text { (days) }\end{array}$ & $\begin{array}{l}\text { Time of } \\
\text { isolation } \\
\text { after } \\
\text { initial } \\
\text { treatment } \\
\text { (days) }\end{array}$ & $\begin{array}{l}\text { Antimicrobial } \\
\text { combination therapy } \\
\text { (days) }\end{array}$ & $\begin{array}{l}\text { Risk } \\
\text { factors }\end{array}$ & $\begin{array}{l}\text { Clinical } \\
\text { outcome } \\
\text { at } 30 \\
\text { days }\end{array}$ & $\begin{array}{l}\text { Mic } \\
\text { out } \\
\text { da) } \\
\text { (da }\end{array}$ \\
\hline 1 & KpBO3 & 0 & 13 & $\begin{array}{l}\text { Abdominal } \\
\text { infection }\end{array}$ & $\begin{array}{l}\text { Ceftazidime/Avibactam } \\
\text { (12), Meropenem (32) }\end{array}$ & 0 & $\begin{array}{l}\text { Meropenem-Colistin- } \\
\text { Tigecycline } \\
\text { (3) }\end{array}$ & - & Failure & NA \\
\hline 2 & KpBO6 & 4 & 2 & Pneumonia & $\begin{array}{l}\text { Meropenem-Colistin (27) } \\
\text { Meropenem-Tigecycline } \\
\text { (19) }\end{array}$ & 0 & $\begin{array}{l}\text { Meropenem-Colistin } \\
(24)\end{array}$ & - & Success & Sur \\
\hline 3 & KpBO7 & 7 & 1 & $\begin{array}{l}\text { CVC- } \\
\text { related }\end{array}$ & $\begin{array}{l}\text { Amoxicillin/Clavulanic } \\
\text { acid (11) }\end{array}$ & 1 & $\begin{array}{l}\text { Meropenem-Colistin } \\
\text { (13) }\end{array}$ & CVVH & Success & Sur \\
\hline 4 & КрВ08 & 60 & 6 & $\begin{array}{l}\text { CVC- } \\
\text { related }\end{array}$ & $\begin{array}{l}\text { Oxacillin (11), } \\
\text { Ertapenem (13) }\end{array}$ & 0 & $\begin{array}{l}\text { Meropenem- } \\
\text { Ceftazidime/Avibactam } \\
(24)\end{array}$ & CKD & Success & Sur \\
\hline 5 & KрВ011 & 18 & 12 & Pneumonia & $\begin{array}{l}\text { Oxacillin (8), } \\
\text { Piperacillin/Tazobactam } \\
\text { (34) }\end{array}$ & 15 & $\begin{array}{l}\text { Meropenem-Colistin } \\
\text { (18) }\end{array}$ & - & Failure & $\begin{array}{l}\text { Fai } \\
(15\end{array}$ \\
\hline 6 & KpB012 & 3 & 20 & $\begin{array}{l}\text { Biliary } \\
\text { infection }\end{array}$ & $\begin{array}{l}\text { Piperacillin/Tazobactam } \\
\text { (3) } \\
\text { Meropenem-Tigecycline } \\
\text { (3) }\end{array}$ & 10 & $\begin{array}{l}\text { Meropenem-Tigecycline } \\
\text { (1) }\end{array}$ & $\begin{array}{l}\text { CKD, } \\
\text { CVVH }\end{array}$ & Failure & NA \\
\hline 7 & KpB013 & 5 & 5 & Pneumonia & $\begin{array}{l}\text { Piperacillin/Tazobactam } \\
\text { (16) } \\
\text { Meropenem-Tigecycline } \\
(29)\end{array}$ & 1 & $\begin{array}{l}\text { Meropenem- } \\
\text { Ceftazidime/Avibactam } \\
(14)\end{array}$ & CKD & Failure & NA \\
\hline 8 & KрВ014 & 60 & 3 & $\begin{array}{l}\text { Urinary } \\
\text { infection }\end{array}$ & $\begin{array}{l}\text { Amoxicillin/Clavulanic } \\
\text { acid (20) } \\
\text { Meropenem (19) }\end{array}$ & 0 & $\begin{array}{l}\text { Meropenem- } \\
\text { Ceftazidime/Avibactam } \\
\text { (14) }\end{array}$ & CKD & Success & Sur \\
\hline
\end{tabular}

Abbreviations: Bloodstream infection, BSI; not changed, NC; Central Venous Catheter, CVC; Continuous Venous Hemofiltration, CVVH; Chronic Kidney Disease, CKD; not applicable, NA.

\section{Supplementary Files}

This is a list of supplementary files associated with this preprint. Click to download.

- Supplementarydata.docx 